\title{
SUBSTITUTIONAL LEMMA FOR G-SPACES OF 1-DIMENSIONAL GROUPS
}

by JUAN ANTONIO PÉREZ

(Received 14 December, 1994)

1. Introduction. Let $G$ be a compact Lie group and $X$ a $G \cdot C W$ complex. We are interested in the calculation of the Borel cohomology of $X$

$$
H_{G}^{*}(X)=H^{*}\left(E G \times_{G} X\right)
$$

where $E G$ is a universal free $G$-space and we use on the right hand side cellular cohomology. For an introduction to $G-C W$ complexes see Matumoto [4] and for a good exposition on Borel cohomology see for instance tom Dieck [2]. We want to replace $X$ with an ordinary $C W$ complex $Y$ in order to find an ordinary $C W$ structure on the Borel construction $E G \times{ }_{G} Y$ so we can use cellular chains to compute the Borel cohomology of $X$. For every compact Lie group one has an extension

$$
1 \rightarrow G_{0} \stackrel{\dot{j}}{\rightarrow} G \stackrel{p}{\rightarrow} \pi_{0}(G) \rightarrow 1,
$$

where $G_{0}$ is the identity component, so for our case $G_{0}$ is isomorphic to the circle group T. We are dealing with the case in which $\pi_{0}(G)$ is isomorphic to $C_{2}$, the cyclic group of order 2. Let us denote by $\tilde{\mathbb{T}}$ the circle group $\mathbb{I} \subset \mathbb{C}$ with $C_{2}$ acting antipodally on it, and analogously, denote $\widetilde{\mathbb{Z}}$. Thus we have the Hochschild cohomology group

$$
H^{2}\left(C_{2}, \tilde{\mathbb{T}}\right) \cong H^{3}\left(C_{2}, \tilde{\mathbb{Z}}\right) \cong \mathbb{Z} / 2
$$

which shows that the only possible extensions of $\mathbb{T}$ by $C_{2}$ where $C_{2}$ acts non-trivially on $\mathbb{T}$ are $O(2)$, the continuous dihedral group and $Q=N_{S U(2)}(\mathbb{T})$, the continuous quaternion group. From now on $G$ will mean either $O(2)$ or $Q$. See Brown [1] for a good exposition on Hochschild cohomology and group extensions.

The same we prove for the mentioned 1-dimensional groups is proved for the circle group in Greenlees and May [3], Lemma 14.1. The point is that the circle group is abelian and connected while $O(2)$ and $Q$ are not. The proof here was inspired by the proof in [3].

2. Preliminary observations. Let us denote by $\mathscr{S}(G)$ the set of closed subgroups of $G$. If we write $H \sim K$ meaning that $H$ and $K$ are conjugate subgroups of $G$, then we can write $\mathscr{C}(G)=\mathscr{S}(G) / \sim$. This provides a projection $\pi: \mathscr{S}(G) \rightarrow \mathscr{C}(G)$ given by $\pi: H \mapsto(H)$. The notation between conjugacy classes $(H)<(K)$ means that the subgroup $H$ is subconjugate to $K$. The first observation is the following.

Lemma 1. Let $G$ be either $O(2)$ or $Q$. If there exists a section $v: \mathscr{C}(G) \rightarrow \mathscr{S}(G)$ such that $(H)<(K)$ then $v((H))<v((K))$.

The existence of a section like the one in Lemma 1 for a general compact Lie group is not clear for the author, furthermore, it is not even clear whether it exists for any finite

Glasgow Math. J. 38 (1996) 215-220. 
group. It would be interesting to find necessary and sufficient conditions for the existence of such a $v$. Now, if $N_{G}(H)$ denotes the normalizer of the closed subgroup $H$ in the group $G$, we will write $W_{G}(H)$ to denote the factor group $N_{G}(H) / H$. Next, the proof of the substitution lemma rests upon the existence of the above $v$ and the existence of the ordinary $\mathrm{CW}$ structures guaranteed by the next observation.

Lemma 2. For $G$ being either $O(2)$ or $Q$, and $H$ any closed subgroup of $G$, there exist ordinary $C W$ structures on $G, G / H$ and $W_{G}(H)$ such that the product on $G$ is a cellular map and for every $H \in \operatorname{Im}(v)$ :

(a) The action of $G$ on $G / H$ is cellular.

(b) $(G / H)^{(0)}=\left\{W_{G}(H)\right\}^{(0)}$.

(c) The group operation in $W_{G}(H)$ is cellular.

(d) For $H<K$ the projection $G / H \rightarrow G / K$ is cellular.

(e) $(G / K)^{H}$ is a subcomplex of $G / K$.

For the sake of brevity, a $C W$ structure like the one in Lemma 2 will be called a good family $C W$ structure in what follows. Also, for the sake of clarity, if $Y$ is an ordinary $C W$ complex, as usual, we denote by $Y^{(k)}$ its ordinary $k$-skeleton while if $X$ is a $G-C W$ complex, its equivariant $k$-skeleton will be written $X^{[k]}$. Section 3 is devoted to state and prove the substitution lemma. In Sections 4 and 5, Lemmas 1 and 2 are proved.

\section{The substitution lemma.}

THEOREM 3. Let $G$ be either $O(2)$ or $Q$. Then for every $G-C W$ complex $X$ there exists a G-CW complex $Y$ which is $G$-homotopy equivalent to $X$ and has a decomposition as an ordinary $C W$ complex with a cellular action by $G$ and such that $Y^{H}$ is a subcomplex of $Y$ for every $H \in \operatorname{Im}(v)$.

Proof. Let $X^{[0]}$ be the equivariant 0 -skeleton of $X$ and define $Y^{[0]}=X^{[0]}$. By regarding $Y^{[0]}$ as a disjoint union of $G$-orbits, the space $Y^{[0]}$ has the structure of an ordinary $C W$ complex given by the $C W$ structure on the homogeneous spaces $G / K$, for $K \in \operatorname{Im}(v)$. Moreover, according to (a) Lemma 2, $G$ acts cellularly on the $C W$ complex $Y^{[0]}$. Note that $\operatorname{dim}\left(Y^{[0]}\right) \leqslant 1$ and thanks to (e) Lemma $2,\left(Y^{[0]}\right)^{H}$ is a subcomplex of $Y^{[0]}$ for every $H \in \operatorname{Im}(v)$. Now, if we take

$$
\xi_{0}: X^{[0]} \rightarrow Y^{[0]}
$$

to be the identity map, we have that $\xi_{0}$ is a $G$-homeomorphism. Assume inductively that we have constructed a $C W$ complex $Y^{[n-1]}$ and a $G$-homotopy equivalence

$$
\xi_{n-1}: X^{[n-1]} \rightarrow Y^{[n-1]},
$$

where $Y^{[n-1]}$ has the structure of an ordinary $C W$ complex with $G$ acting cellularly on it and such that $\left(Y^{[n-1]}\right)^{H}$ is a subcomplex of $Y^{[n-1]}$ for any $H \in \operatorname{Im}(v)$. Let us take an equivariant $n$-cell of $X$ with domain $(G / H) \times E^{n}$, and denote by $\varphi$ the corresponding attaching map and by $\varphi^{0}$ its restriction to $(G / H)^{(0)} \times S^{n-1}$. To carry on, we need to make the following observation which will be proved in a final section.

Lemma 4. The 0-skeleton $(G / H)^{(0)}$ has a group structure making the sub-complex $\left(Y^{[n-1]}\right)^{H} a(G / H)^{(0)}$-space. 
Using the equivariant cellular approximation theorem, let $\gamma$ be a $(G / H)^{(0)}$-cellular approximation to the following composite

$$
(G / H)^{(0)} \times S^{n-1} \stackrel{\varphi^{0}}{\longrightarrow}\left(X^{[n-1]}\right)^{H} \stackrel{\epsilon_{n-1}^{H}}{\longrightarrow}\left(Y^{[n-1]}\right)^{H}
$$

The following two lemmas are also proved in the last section.

Lemma 5. The map $\Gamma:(G / H) \times S^{n-1} \rightarrow Y^{[n-1]}$ defined by $\Gamma(g H, x)=g \gamma(H, x)$ is an extension of $\gamma$ and it is $G$-equivariant.

Lemma 6. The map $\Gamma$ defined in the above lemma is cellular.

Now that $\Gamma$ is cellular, the mapping cone $Y^{[n-1]} \cup_{\Gamma}\left\{(G / H) \times E^{n}\right\}$ is an ordinary $C W$ complex and also, given any $K<G$, we have

$$
\left[Y^{[n-1]} \cup_{\Gamma}\left\{(G / H) \times E^{n}\right\}\right]^{K}=\left(Y^{[n-1]}\right)^{K} \cup_{\Gamma^{K}}\left\{(G / H)^{K} \times E^{n}\right\}
$$

which is also a $C W$ complex by the inductive hypothesis and Lemma 6 . Taking this $\Gamma$ as a typical attaching map for the construction of $Y^{[n]}$, we get an ordinary $C W$ structure for $Y^{[n]}$, where the $G$-action is cellular and $\left(Y^{[n]}\right)^{H}$ is a subcomplex for every $H<G$.

Finally, by comparison of cofiber sequences as shown in the following diagram

$$
\begin{aligned}
& G / H \times S^{n-1} \stackrel{\varphi}{\longrightarrow} Y^{[n-1]} \stackrel{\subset}{\longrightarrow} Y^{[n-1]} \cup_{\Gamma}\left\{(G / H) \times E^{n}\right\} \\
& \text { । }\|\|_{1} \quad \zeta_{n-1} \mid \llbracket \xi_{n-1} \quad \zeta_{n-1} \cup 1 \downarrow \| \xi_{n-1} \cup 1 \\
& G / H \times S^{n-1} \stackrel{r}{\longrightarrow} Y^{[n-1]} \stackrel{\subset}{\longrightarrow} Y^{[n-1 \mid} \cup_{\Gamma}\left\{(G / H) \times E^{n}\right\}
\end{aligned}
$$

we see that $\xi_{n-1}$ and its homotopy inverse $\zeta_{n-1}$ extend cell by cell to a $G$-homotopy equivalence

$$
\xi_{n}: X^{[n]} \rightarrow Y^{[n]}
$$

and its inverse $\zeta_{n}$ respectively. This completes the induction and proves the theorem.

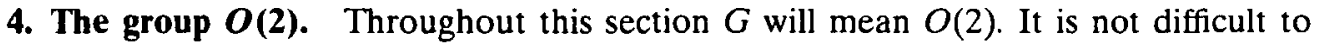
prove that a proper closed subgroup $H<G$ is conjugate to either $\mathbb{T}, C_{n}$ or $D_{2 n}$, the dihedral group of order $2 n$. Here, we identify the circle group $\pi$ with the identity component $S O(2)$ of $O(2), C_{n}<\pi$ and $D_{2 n}$ has underlying set $C_{n} \cup r C_{n}$ for

$$
r=\left[\begin{array}{rr}
1 & 0 \\
0 & -1
\end{array}\right] \in O(2)
$$

Therefore, for this case, the diagram of conjugacy classes of subgroups looks as follows

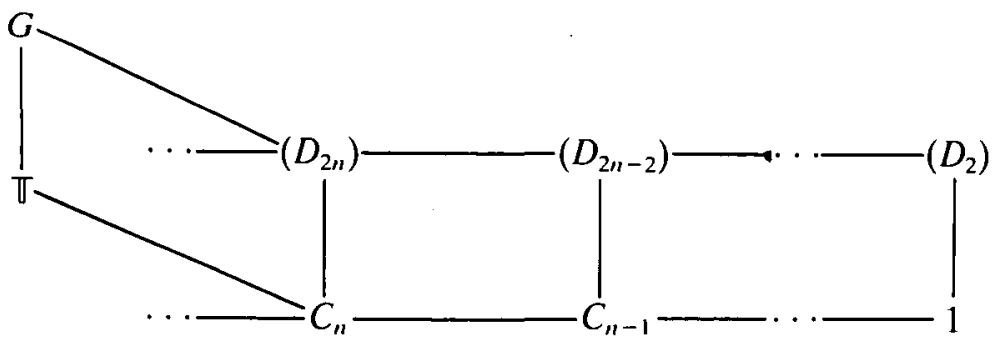


and to define the required good section $v$ it is enough to give its image

$$
\operatorname{Im}(v)=\{G, \mathbb{T}\} \cup\left\{D_{2 n} \mid n \in \mathbb{Z}\right\} \cup\left\{C_{n} \mid n \in \mathbb{Z}\right\} .
$$

The $G$-orbits are either a discrete space having at most two points or a collection of at most two circles. Let us give to $G$ a $C W$ structure with $G^{(0)}=\{1, r\}$ and two 1-cells in the obvious way, which makes cellular the operation in $G$. Since if $H$ is not a non-trivial finite cyclic subgroup then $W_{G}(H)$ is a finite set, so define $(G / H)^{(0)}=W_{G}(H)$. If $H$ is cyclic then $W_{G}(H)=G / H$, for this case define $(G / H)^{(0)}=\left[W_{G}(H)\right]^{(0)}=\{H, r H\}$.

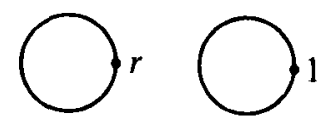

5. The group $Q$. The continuous quaternion group $Q=N_{s U(2)}(\mathbb{T})$ may be seen as consisting of the matrices of $S U(2)$ having the forms

$$
\left[\begin{array}{cc}
e^{i \theta} & 0 \\
0 & e^{-i \theta}
\end{array}\right] \text { and }\left[\begin{array}{cc}
0 & e^{i \theta} \\
-e^{-i \theta} & 0
\end{array}\right]
$$

We have seen that $Q=N_{S U(2)}(\mathbb{T})$, where $\pi$ means now the maximal torus $U(1) \subset S U(2)$. The group $Q$ is generated by $\mathbb{T}$ and the element

$$
j=\left[\begin{array}{rr}
0 & 0 \\
-1 & 0
\end{array}\right]
$$

which clearly satisfies $j^{2}=-1$ and $j e^{i \theta_{j}-1}=e^{-i \theta}$. Using the equality $e^{i(\pi / 2)}=i$ we introduce the symbol $k=i j$. After that, we can recognize the quaternion group $Q_{8}$ with underlying set $\{ \pm 1, \pm i, \pm j, \pm k\}$ as a subgroup of $Q$. Now, a generalized quaternion group (see [1], p. 98), denoted $Q_{4 n}$ is defined to be the subgroup of $Q$ generated by the normal subgroup $C_{2 n}<\pi$ and the element $j$, for $n \geqslant 1$. The group $Q_{4 n}$ has order $4 n$ and we note that $i, k \in Q_{4 n}$ if $n$ is even and $i, k \in Q-Q_{4 n}$ for $n$ odd. For a start, we need to characterize the closed subgroups of $Q$. It is easy to check that a closed proper subgroup $H<Q$ is isomorphic to either T, $C_{n}$ or $Q_{4 n}$, so we get the following diagram of conjugacy classes.

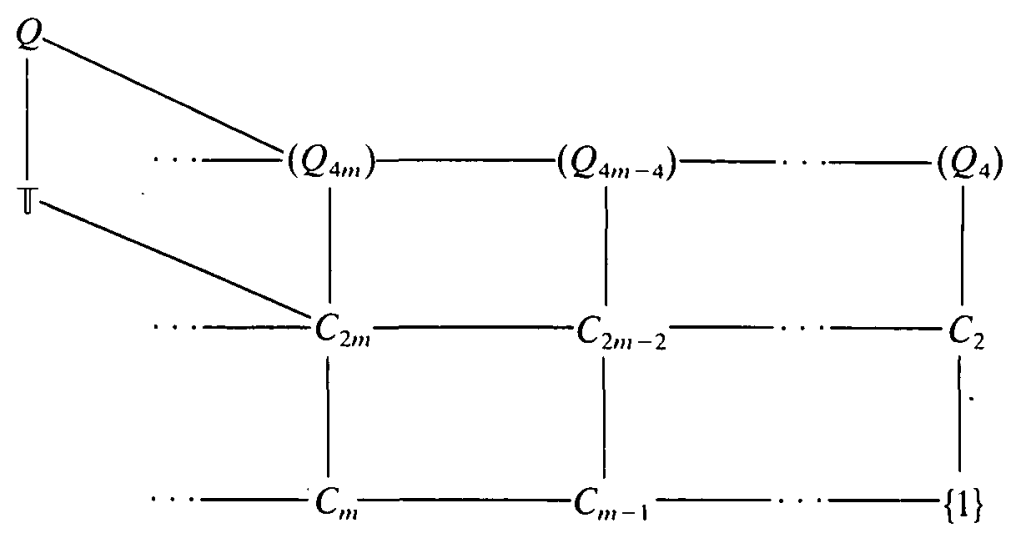

Again, in order to define the good section $v$ it suffices to give its image

$$
\operatorname{Im}(v)=\{Q, \mathbb{T}\} \cup\left\{Q_{4 n} \mid n \in \mathbb{Z}\right\} \cup\left\{C_{n} \mid n \in \mathbb{Z}\right\} .
$$


As in the case of $O(2)$ the $Q$-orbits are either discrete with at most two points or a collection of at most two circles. Let us give to $Q$ a $C W$ structure with $Q^{(0)}=\{ \pm 1, \pm j\}$ and four 1-cells in the obvious way, so the operation in $Q$ becomes cellular. If $H$ is not a non-trivial finite cyclic subgroup then $W_{Q}(H)$ is a finite set, so we may define $(G / H)^{(0)}=W_{Q}(H)$. If $H$ is nontrivial finite cyclic having even order, since $-1 \in H$, this shows that we need to make $(G / H)^{(0)}=\{H, j H\}$; alternatively, if $H$ has odd order, we need $(G / H)^{(0)}=\{ \pm H, \pm j H\}$.

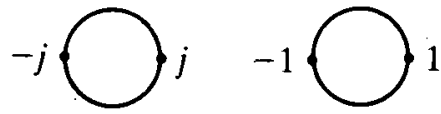

6. Remaining proofs. In this section, $G$ will mean either $O(2)$ or $Q$. It is straightforward to verify that the $C W$ structures given in the last two sections satisfy (a)-(d) in Lemma 2. To verify (e) we just need to observe that for $H<K$ one has $(G / K)^{H}=\operatorname{Map}^{G}(G / H, G / K) \cong \mathbb{Z} / 2$ for the case in which neither $H$ nor $K$ are normal in $G$. If one of them is normal then $(G / K)^{H}=G / K$. Next we give here proofs of some facts that were used in the proof of the substitution lemma. It is worth remarking that the above cell structures make the 0 -skeleton of $G / H$ into a subgroup of $W_{G}(H)$. All the references in the following proofs are related to Lemma 2.

Proof of Lemma 4. From (d), the projection $G \rightarrow G / H$ is cellular, so $H \in(G / H)^{(0)}$, and also $H \in W_{G}(H)^{(0)}$ by (b). By (c) we have $W_{G}(H)^{(0)}<W_{G}(H)$ so $(G / H)^{(0)}<W_{G}(H)$.

Proof of Lemma 5. First of all, it is clear that $\Gamma$ extends $\gamma$, since for $g H \in(G / H)^{(0)}$ we have

$$
\Gamma(g H, x)=g \gamma(H, x)=g H \gamma(H, x)=\gamma(g H, x)
$$

recalling that $\gamma$ is a $(G / H)^{(0)}$-map. Now, for any $g \in G$ we have

and also, since $H \in(G / H)^{(0)}$,

$$
\Gamma(g H, x)=g \gamma(H, x)
$$

$$
\Gamma(g H, x)=g \gamma(H, x)=g \Gamma(H, x)
$$

which proves $G$-equivariance.

Proof of Lemma 6. Recall that we want to prove the cellularity of our new $\Gamma$. It is enough to do it for $(G / H)^{(0)} \times\left(S^{n-1}\right)^{(n-1)}$ and for $(G / H)^{(1)} \times\left(S^{n-1}\right)^{(0)}$, since by dimensional reasons the other products take care of themselves.

First, the restriction of $\Gamma$ to $(G / H)^{(0)} \times\left(S^{n-1}\right)^{(n-1)}$ is $\gamma$, and it was chosen to be cellular, so the given product is mapped by $\Gamma$ to the ordinary $(n-1)$-skeleton of $Y^{[n-1]}$.

By our definition of $\Gamma$, the product $(G / H)^{(1)} \times\left(S^{n-1}\right)^{(0)}$ is mapped by $\Gamma$ to the orbit $G(\Gamma(H, *))=G(\gamma(H, *))$. We have that $\gamma(H, *)$ maps into the ordinary 0-skeleton of $Y^{[0]}$ and this clearly means $G(\gamma(H, *)) \subseteq Y^{[0]}$. Use now the cellularity of the projections.

\section{REFERENCES}

1. K. S. Brown, Cohomology of groups (Springer Verlag, 1982).

2. T. tom Dieck, Transformation groups. (Walter de Gruyter, 1987). 
3. J. P. C. Greenlees and J. P. May, Generalized Tate cohomology, Mem. Amer. Math. Soc. (to appear).

4. T. Matumoto, On G.CW complexes and a theorem of J. H. C. Whitehead, J. Fac. Sci. Univ. Tokio 18 (1971) 363-374.

Centro Regional de Estudios Nucleares

Universidad Autónoma de Zacatecas

APARTADO POSTAL 495

98068 ZaCATECAS, ZAC.

MÉxıco

email; japerez@bufa.cantera 